\title{
Reverse transcription using random pentadecamer primers increases yield and quality of resulting cDNA
}

\author{
Michael Stangegaard ${ }^{1}$, Inge Høgh Dufva ${ }^{2}$, and Martin Dufva ${ }^{1}$
}

BioTechniques 40:649-657 (May 2006)

doi 10.2144/000112153

\begin{abstract}
Reverse transcription of RNA is an invaluable method for gene expression analysis by real-time PCR or microarray methods. Random primers of varying lengths were compared with respect to their efficiency of priming reverse transcription reactions. The results showed that 15-nucleotide-long random oligonucleotides (pentadecamers) consistently yielded at least 2-fold as much cDNA as did random hexamers using either poly(A) RNA or an amplified version of messenger RNA (aRNA) as a template. The cDNA generated using pentadecamers did not differ in size distribution or the amount of incorporated label compared with cDNA generated with random hexamers. The increased efficiency of priming using random pentadecamers resulted in reverse transcription of $>80 \%$ of the template aRNA, while random hexamers induced reverse transcription of only $40 \%$ of the template aRNA. This suggests a better coverage of the transcriptome when using random pentadecamers over random hexamers. Using the same amount of aRNA as starting material, random pentadecamer-primed reactions resulted in 11-fold more genes being detected in whole transcriptome $D N A$ microarray experiments than random hexamer-primed reactions. The results indicate that random pentadecamers can replace random hexamers in reverse transcription reactions on both poly(A) RNA and amplified RNA, resulting in higher cDNA yields and quality.
\end{abstract}

\section{INTRODUCTION}

Reverse transcription of RNA into cDNA is a core method for analysis of gene expression using DNA microarray or real-time PCR (1-5). The reverse transcription should result in a cDNA population that reflects the original messenger RNA (mRNA) population in terms of transcript abundance and complexity. Furthermore, the reverse transcription reaction should be as efficient as possible to give maximum sensitivity in the final assay. Sensitivity is often problematic in microarray analysis of unamplified mRNA. Sensitivity is also an issue when following fusion gene markers in cancer patients where, for instance, it is desired to detect a single cancer cell expressing the BCR/ABL fusion transcripts in a background of 100,000 normal cells in chronic myeloid leukemia (CML) patients $(6,7)$. This suggests that improvements in reverse transcription could have large impact in assay results.

RNA quality (8-12), priming strategy (13), and enzyme efficiency $(14,15)$ are important parameters for obtaining high yield cDNA of good quality. Less studied is the impact of different primers in the reaction. Reverse transcription reactions can be primed using specific primers $(16,17)$ if relatively few mRNA species are targeted. This approach is not practical for whole transcriptome analysis using microarray, because it would require synthesis and mixing of thousands of specific primers. In those cases, the reverse transcription reaction is primed with oligo(dT) $(18,19)$ random hexamers $(18,20-23)$ or random nonamers $(23,24)$. Oligo(dT) priming has the virtue of producing cDNA from the $3^{\prime}$ end of poly(A) mRNA, allowing total RNA to be used as a template. The drawback is that oligo(dT) priming often results in a $3^{\prime}$ bias compared with random priming (14). Poly(A)-selected RNA like an isolated mRNA fraction or amplified RNA (aRNA) are preferably reverse transcribed with random primers, because random priming is less likely to give a $3^{\prime}$ end bias in the resulting cDNA.

Here we report a novel priming method for reverse transcription reactions and a study of the implication of random primer length in reverse transcription reactions on the yield of the resulting cDNA and the influence on the amount of detectable genes on oligonucleotide microarrays.

\section{MATERIALS AND METHODS}

\section{Cells}

Human carcinoma cells (HeLa) were cultured in $75 \mathrm{~cm}^{2}$ culture flasks (EasyFlask ${ }^{\mathrm{TM}}$; Nalge Nunc International, Rochester, NY, USA) in

\footnotetext{
${ }^{1}$ Technical University of Denmark, Lyngby and 2University of Copenhagen, Herlev, Denmark
} 
$25 \mathrm{~mL}$ RPMI 1640 media supplemented with $10 \%$ fetal bovine serum (FBS), penicillin $(100 \mathrm{U} / \mathrm{mL})$, and streptomycin $(100 \mu \mathrm{g} / \mathrm{mL}$; all from Sigma, St. Louis, MO, USA). Cells were cultured at $37^{\circ} \mathrm{C}$ in an atmosphere containing $5 \% \quad \mathrm{CO}_{2} \quad(\mathrm{AGA}, \quad$ Copenhagen, Denmark) in a $\mathrm{CO}_{2}$ incubator (Assab; Don Whitley Scientific Ltd., Shipley, West Yorkshire, UK). The cells were seeded at a density of 6667 cells $/ \mathrm{cm}^{2}$, and the cells were subcultured every 3 to 4 days when approximately $90 \%$ confluence was reached.

\section{RNA Isolation and Amplification}

Total RNA was isolated by using RNeasy $^{\circledR}$ Mini Kit spin columns (Qiagen, Valencia, CA, USA). Quantification of total RNA was performed in an Ultraspec 3000 spectrophotometer (Pharmacia Biotech, Cambridge, UK), and validation of RNA quality was performed by using the RNA 6000 Nano Assay on an Agilent 2100 Bioanalyzer (Agilent Technologies, Palo Alto, CA, USA). Amplification was performed with the RiboAmp ${ }^{\circledR}$ T7-based RNA amplification kit (Arcturus Engineering, Mountain View, CA, USA) according to the manufacturer's instructions using $2 \mu \mathrm{g}$ total RNA as a starting material, yielding 35-40 $\mu \mathrm{g}$ aRNA after one round of amplification. For each labeling reaction, $3 \mu \mathrm{g}$ aRNA were used unless otherwise mentioned. The mRNA fraction was isolated from $100 \mu \mathrm{g}$ total RNA using oligo(dT) ${ }_{25}$ Dynabeads $^{\circledR}$ (Dynal, Oslo, Norway) according to the manufacturer's instructions. The mRNA $(2-3 \mu \mathrm{g})$ was eluted in $20 \mu \mathrm{L}$ buffer $(10 \mathrm{mM}$ Tris- $\mathrm{HCl}, \mathrm{pH}$ 7.5).

\section{cDNA Synthesis}

Synthesis of cDNA was performed as described elsewhere (23), with the following modifications. In brief, 2-3 $\mu \mathrm{g}$ aRNA were mixed with $3.35 \mathrm{nmol}$ random primers (Sigma-Genosys, Haverhill, UK or Invitrogen, Paisley, UK), unless otherwise mentioned, in a total volume of $18.5 \mu \mathrm{L}$. The mixture was heated at $70^{\circ} \mathrm{C}$ for 10 min and snap-cooled on ice for at least $1 \mathrm{~min}$. Reverse transcription reaction mixture lacking enzyme was added to a final concentration of 500 $\mu \mathrm{M}$ each dATP, dCTP, and dGTP, $300 \mu \mathrm{M}$ dTTP (Larova Biochemie GMBH, Teltow, Germany), $200 \mu \mathrm{M}$ 5-aminoallyl-dUTP (Sigma-Aldrich, Steinheim, Germany), 40 U RNasin ${ }^{\circledR}$ (Promega, Mannheim, Germany), 1× first-strand buffer (Invitrogen), $10 \mathrm{mM}$ dithiothreitol (DTT; Invitrogen) in a final volume of $29 \mu \mathrm{L}$. The reaction mixture was incubated for 3-5 min at room temperature to let the primers anneal. SuperScript ${ }^{\mathrm{TM}}$ II (400 U, $2 \mu \mathrm{L}$; Invitrogen) were added, and the mixture was incubated at $42^{\circ} \mathrm{C}$ for $5 \mathrm{~h}$. Avian myeloblastosis virus (AMV)-catalyzed reactions were performed in $100-\mu \mathrm{L}$ reactions containing $3 \mu \mathrm{g}$ aRNA, $1 \times$ reaction buffer, $6 \mu \mathrm{g}$ either Inv or R15 primer, $60 \mathrm{U}$ RNasin, $50 \mu \mathrm{g} / \mathrm{mL}$ BSA, $500 \mu \mathrm{M}$ each dATP, dCTP, and dGTP, $300 \mu \mathrm{M}$ dTTP, $200 \mu \mathrm{M}$ 5-aminoallyldUTP, and 40 U AMV enzyme (USB, Cleveland, Ohio, USA). Moloney murine leukemia virus (MmLV)catalyzed reactions were performed in $50-\mu \mathrm{L}$ reactions containing $3 \mu \mathrm{g}$ aRNA, $6 \mu \mathrm{g}$ either Inv or R15 primer, $1 \times$ reaction buffer, $40 \mathrm{U}$ RNasin, 500 $\mu \mathrm{M}$ each dATP, dCTP, and dGTP, 300 $\mu \mathrm{M}$ dTTP, $200 \mu \mathrm{M}$ 5-aminoallyl-dUTP, and $500 \mathrm{U}$ MmLV enzyme (USB). Annealing of primers was performed identically to SuperScript II-catalyzed reactions, and the enzymes were added last to all reactions.

Reactions were terminated by the addition of $10 \mu \mathrm{L} 0.5 \mathrm{M}$ EDTA (Sigma), and the RNA was hydrolyzed by the addition of $10 \mu \mathrm{L} 1 \mathrm{M} \mathrm{NaOH}$ (Sigma) followed by heating at $65^{\circ} \mathrm{C}$ for $15 \mathrm{~min}$. Reactions were neutralized by adding $10 \mu \mathrm{L} 1 \mathrm{M} \mathrm{HCl}$ (Sigma). The cDNA was subsequently purified on QIAquick ${ }^{\circledR}$ PCR Purification kit spin columns (Qiagen) according to the manufacturer's instructions, but substituting phosphate wash buffer [5 $\mathrm{mM}$ potassium phosphate (Sigma), $\mathrm{pH} 8.0,80 \%$ ethanol] for buffer PE and phosphate elution buffer $(4 \mathrm{mM}$ potassium phosphate, $\mathrm{pH}$ 8.5) for buffer EB.

The cDNA was lysophilized and resuspended in $4.5 \mu \mathrm{L} 0.1 \mathrm{M}$ fresh sodium carbonate buffer, $\mathrm{pH}$ 9.0. Nhydroxysuccinimide (NHS) esters (4.5 $\mu \mathrm{L}$ ) of $\mathrm{Cy}^{\mathrm{TM}} 3$ or $\mathrm{Cy} 5$ dye (Amersham
Biosciences, Piscataway, NJ, USA) in dimethylsulfoxide (DMSO, lysophilized dye from one tube was dissolved in $73 \mu \mathrm{L}$ DMSO; Sigma) were added, and reactions were incubated at room temperature in the dark for $1 \mathrm{~h}$. Coupling reactions were quenched by the addition of $35 \mu \mathrm{L} 0.1$ M sodium acetate (Sigma), pH 5.2, and unincorporated dye was removed using QIAquick PCR Purification kit spin columns according to the manufacturer's instructions. Labeling efficiency was determined by analyzing the whole undiluted sample in a spectrophotometer using a 50$\mu \mathrm{L}$ microcuvette (Hellma, Müllheim, Germany).

\section{Microarray}

Slides prespotted with 44,290 60-nucleotide-long oligonucleotides directed against the human transcriptome were obtained (Agilent Technologies), and hybridizations were performed according to supplier's instructions. In brief, unless otherwise mentioned, 200 pmol of each of the differently Cy-labeled cDNA reactions were combined in $200 \mu \mathrm{L}$ Milli- ${ }^{\circledR}$ water (Millipore, Billerica, MA, USA) and denatured at $98^{\circ} \mathrm{C}$ for 3 min followed by incubation at room temperature for 2-3 min. Fifty microliters $10 \times$ control (Agilent) and $250 \mu \mathrm{L}$ $2 \times$ hybridization buffer (Agilent) were then added to the cDNA mixture. Four hundred ninety microliters were applied to a gasket slide (Agilent) placed in a hybridization chamber (Agilent). The slide containing the microarray was mounted on top of the gasket slide containing the hybridization solution, and the hybridization chamber was then closed and inspected for bubble formation. Stationary bubbles were dislodged by gentle tapping. The hybridization chamber was mounted on a rotation device and placed in a Hybaid Shake 'n' Stack hybridization oven (Thermo Electron Corporation, San Diego, CA, USA) for $17 \mathrm{~h}$ at $60^{\circ} \mathrm{C}$ with the lowest rotation setting (approximately $4 \mathrm{rpm}$ ).

Following hybridization, the gasket slide was removed from the slide containing the array while submerged in washing solution 1 [ $6 \times$ standard saline 


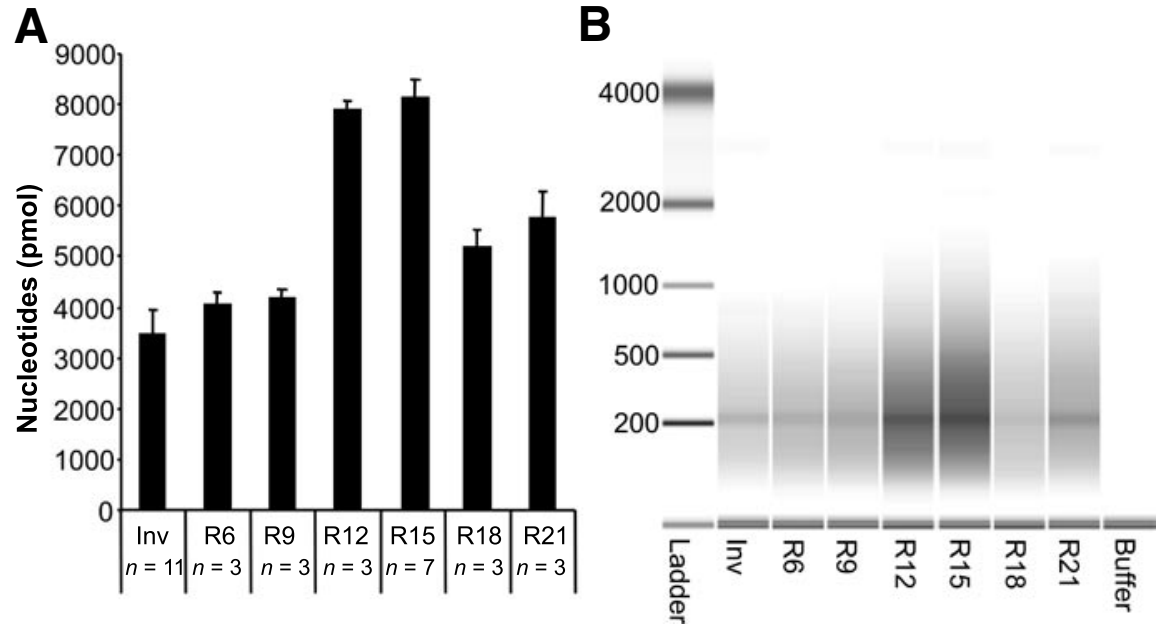

Figure 1. Effect of random primer length on the amount of generated cDNA. (A) cDNA yield obtained from reverse transcription of $3 \mu \mathrm{g}$ amplified RNA (aRNA). Values are based on spectrophotometer measurements $\left(A_{260}-A_{310} \mathrm{~nm}\right)$. A commercial random primer formulation primarily consisting of random hexamers from Invitrogen (Inv) was included as a control. Random primers were obtained from Sigma Genosys ranging in length from 6 to 21 bases using a 3 base length increment (R6 to R21). (B) Size distribution of the generated cDNA using the 2100 Bioanalyzer and the RNA 6000 Nano LabChip ${ }^{\circledR}$ (Agilent).

citrate (SSC; AppliChem, Darmstadt, Germany), $0.005 \%$ Triton ${ }^{\circledR} \mathrm{X}-102$ (Agilent)]. The slides were washed for $10 \mathrm{~min}$ at room temperature in washing solution 1 followed by 5 min in wash solution $2(0.1 \times \mathrm{SSC}, 0.005 \%$ Triton $\mathrm{X}-102)$ at $4^{\circ} \mathrm{C}$ in an in-house fabricated microarray washing station. The slides were dried by centrifugation at $100 \times g$ and scanned in an arrayWoRx ${ }^{\mathrm{TM}}$ microarray scanner (Applied Precision, Issaquah, WA, USA). Quantification was performed in the quantification software accompanying the arrayWoRx scanner.

\section{Data Analysis}

The data was normalized using the Qspline normalization method (25), and further analysis was performed using Excel $^{\circledR}$.

\section{RESULTS}

\section{Yield of cDNA Synthesis Using Different Primers}

cDNA reactions (23) were primed with random primers, ranging from 6 bases to 21 bases in length, to evaluate the effect of primer length on the yield and quality of the resulting cDNA. As control, the Invitrogen random primer
R6 and R9 primers obtained from an oligonucleotide vendor results in cDNA yields comparable to reactions initiated with the commercial random primer formulation, Inv. Because the reactions were spiked with $3 \mu \mathrm{g}$ T7amplified RNA, the maximum yield was 10,000 pmol synthesized nucleotides of cDNA. Reactions initiated with R15 and R12 resulted in $80 \%-90 \%$ yield, while R6- and R9-initiated reactions resulted in $40 \%$ yield. The relative yield between different priming methods was verified by electrophoresis, where R15 primers gave significantly more cDNA than the other primers (Figure 1B). The gel image also suggests that all unused primers were removed during purification and therefore did not contribute to the resulting cDNA yields measured by spectroscopy. There was no difference in size distributions of the respective cDNA populations produced by different primers. Synthesis initiated using different primers did not affect the amount of aminoallyl-dUTP incorporated in the cDNA, as all cDNA had a ratio of nucleotides per dye (Nuc/Dye) that was approximately $40 \pm 15$ (Figure 2).

Reverse transcription of mRNA was investigated, and the results showed that R15 primer-initiated reactions yielded 2-fold more cDNA than reactions initiated with Inv primers, confirming the findings from reactions with aRNA as the template (Figure 1). The results also showed that R15-initiated reactions reverse transcribed almost $80 \%$ of mRNA, which was 2-fold better than the Invitrogen random primer-initiated reactions used as control.

Adding a C6dT modification to random hexamer primers has previously been shown to increase the sensitivity of microarray assay 10 -fold (22). However, in our hands, a random hexamer primer modified with a primary amine attached by a $\mathrm{C} 6$ linker to the $5^{\prime}$ end did not give more cDNA in reverse transcription of mRNA compared with corresponding unmodified primers. No positive effect in the amount of incorporated dye in the cDNA was observed using aminomodified random hexamers compared with unmodified primers (data not shown). 


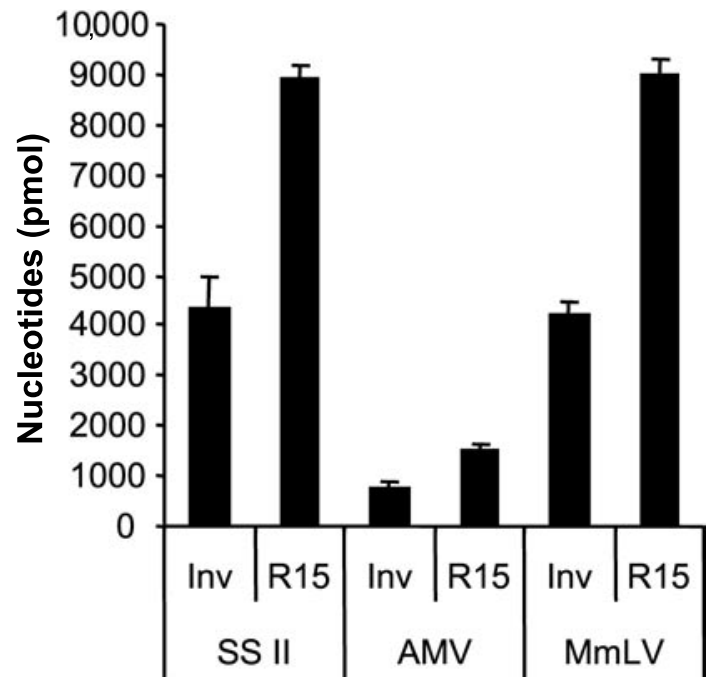

Figure 3. Yield of cDNA synthesis using different reverse transcriptase enzymes. All reactions were initiated with $6 \mu \mathrm{g}$ primer and $3 \mu \mathrm{g}$ amplified RNA (aRNA) originating from the same pool. All reactions were performed at $42^{\circ} \mathrm{C}$ for $5 \mathrm{~h}$. SS II, SuperScript II; AMV, avian myeloblastosis virus; MmLV, Moloney murine leukemia virus.

\section{Yield of cDNA Using Different Polymerases}

The effect of different reverse transcription polymerases on the yield of cDNA was investigated and showed that for all three enzymes used, a 2fold increase in yield was obtained using random pentadecamer primers compared with reactions primed with random hexamer primers (Figure 3). The SuperScript II and the MmLV were found to have close to identical yields (about 90\%), while the AMV polymerase had a significantly lower yield.

\section{Microarray Analysis of cDNA Populations}

The R15 primergenerated cDNA populations were compared with cDNA populations generated by Inv priming by co-hybridization of the respective cDNA populations to DNA microarrays of 44,000 probes. All cDNA populations were generated using equal amounts of the same aRNA pool as template. Genes were considered to be differently expressed significantly if the ratio between the R15- and Inv-primed cDNA exceeded 2-fold as indicated by the upper and lower line on Figure 4, A-C. Repeating the experiments with the order of the dyes reversed enabled removal on nonsignificant noise and statistical analysis of the data. The results showed that, statistically, there were only three gene transcripts that were found to differ between the R15and Inv-primed reactions (Figure 4, A and $\mathrm{B}$, and Table 1). In comparison, cDNA populations generated from four different Inv-primed reactions differed statistically in two transcripts (Figure 4C and Table 1).
Inspecting the non-normalized microarray data showed that $55 \%-72 \%$ more genes were detected using cDNA generated by R15 priming compared with cDNA generated by Inv priming (see Figure 5, A, B, and F, two leftmost bars). This result cannot be explained by differences in fluorescence or amount of target hybridized between the two populations because the amount of dye was normalized to $200 \mathrm{pmol}$ dye prior to hybridization. The effect of increasing signals was highest in the $\mathrm{Cy} 5$ channel (Figure 5F), which can be explained by the fact that Cy5 signals were generally weaker than $\mathrm{Cy} 3$ signals. Normalization of the data removed the positive effect of R15 priming (Figure 4, A and B, and Supplementary Figure S1 available online at www.BioTechniques.com), which is expected because the algorithm (25) is designed to remove systematic variations. We routinely obtain twice as much cDNA during reverse transcription with R15 priming over Inv/R6 priming (Figure 1). The significance of that was illustrated by co-hybridizing microarrays with twice the amount of R15primed cDNA as Inv-primed cDNA. Between 3- and 11-fold more genes were considered expressed on arrays hybridized with twice the amount of R15-primed cDNA compared with Invprimed cDNA (Figure 5, C, D, and F). This means that from the same amount of starting material, cDNA generated by $\mathrm{R} 15$ priming results in more genes being

Table 1. Genes Showing Differences in Expression in cDNA Populations Primed with Either R15 or Inv Primers

\begin{tabular}{|c|c|c|c|c|c|c|}
\hline Probe & Gene & Ratio & SD & $\begin{array}{l}c v \\
(\%)\end{array}$ & $P$ Value & Sequence \\
\hline A_23_P355447 & ZDHHC22 & $0.48^{a}$ & 0.01 & 1.86 & 0.0002 & $\begin{array}{l}\text { 5'-CAATGGGAGGGAGAGGGAGGAGGGGAAGA-3' } \\
\text { 5'-TCTGGGCAATTTTGGCCTTGACTCTTTCCTG-3' }\end{array}$ \\
\hline A_32_P158433 & THC2001391 & $2.20^{a}$ & 0.29 & 13.37 & 0.029 & $\begin{array}{l}\text { 5'-CAGAAGTCGCTGGGCTCATAAGGCTCTTAG-3' } \\
\text { 5'-ACGTGCTTGAGAGTGAGCCTTTCGAAGAGA-3' }\end{array}$ \\
\hline A_32_P187875 & A_32_P187875 & $2.12^{a}$ & 0.15 & 6.93 & 0.0085 & $\begin{array}{l}\text { 5'-GAAGCATCGTATCACAGCAGGTTACAACAA-3' } \\
\text { 5'-CTTTGGGATAAAAGGCAACTGGTAAACTGT-3' }\end{array}$ \\
\hline A_23_P87879 & CD69 & $0.08^{b}$ & 0.04 & 48.58 & 0.00079 & $\begin{array}{l}\text { 5'-TGTGCAATATGTGATGTGGCAAATCTCTATT-3' } \\
\text { 5'-AGGAAATATTCTGTAATCTTCAGACCTAG-3' }\end{array}$ \\
\hline A_24_P694760 & AK056312 & $2.26^{b}$ & 0.29 & 12.65 & 0.025 & $\begin{array}{l}\text { 5'-TCAACTTTGCCTTGATAATTATTGTAAACAC-3' } \\
\text { 5'-TTTGTTCATTTTTTCTTTTTTATTCACAA-3' }\end{array}$ \\
\hline \multicolumn{7}{|c|}{$\begin{array}{l}\text { Genes showing significant differences in expression in cDNA populations primed with either R15 or Inv primers using equal amounts of the same amplified RNA } \\
\text { (aRNA) pool as template (Figure 4, A-B). The ratio of Inv R6 over R15 (a) was calculated for two slides, and the values were averaged. The } P \text { values were calcu- } \\
\text { lated using the Student's } t \text {-test. Genes were considered significantly regulated if the fold-change exceeded } 2 \text {-fold and the } P \text { value was less than } 0.05 \text {. The Inv R6 } \\
\text { over Inv R6 ratios were calculated by utilizing the Inv R6 values from three slides and averaging the ratios. Thus, inherent slide-to-slide as well as experimental } \\
\text { variations are contained in these values. } \\
\text { aRatio refers to the ratio Inv R6 over R15. } \\
\text { bRatio refers to the average ratio values over three slides for Inv R6 signals. }\end{array}$} \\
\hline
\end{tabular}



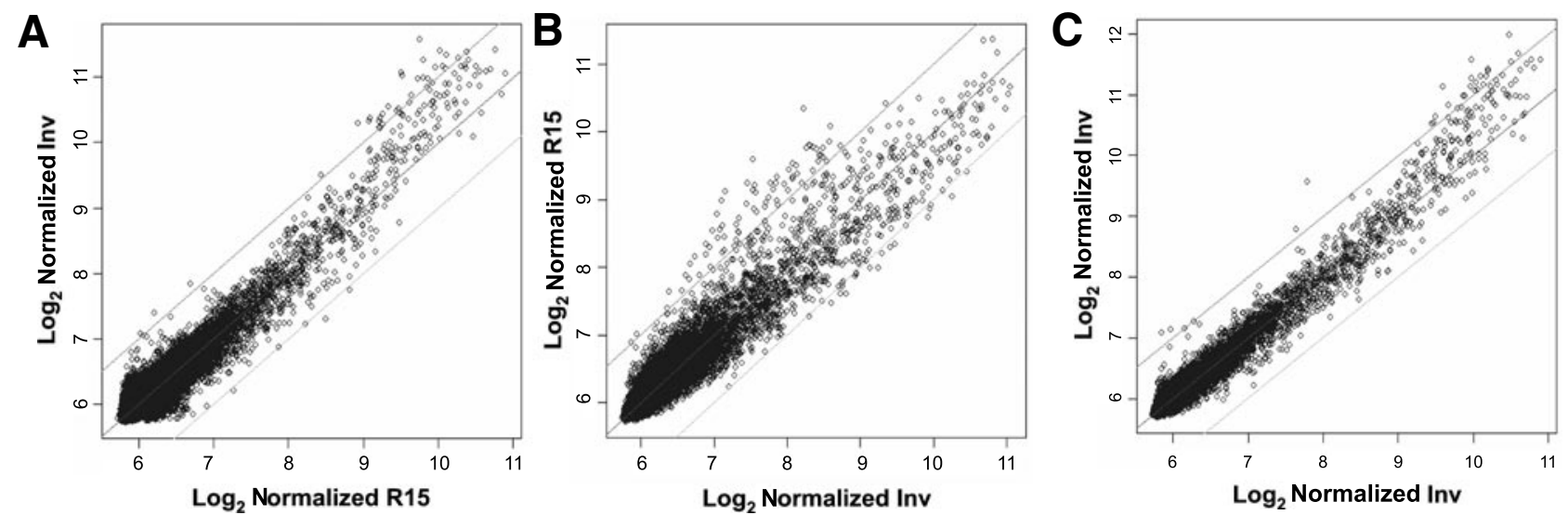

Figure 4. Scatter plots of normalized data from hybridizations to whole human transcriptome arrays. Cy5-labeled samples were always plotted on the y-axis. Prior to hybridization, the differently labeled cDNA samples were pooled using equimolar amounts of each Cy dye (200 pmol). Molar amounts mentioned in pmol in this figure refer to the amount of Cy dye incorporated in the cDNA and not the amount of nucleotides of cDNA. (A) Normalized scatter plot of co-hybridization of 200 pmol Cy5-labeled cDNA resulting from reactions initiated with Inv primes and 200 pmol Cy3-labeled cDNA resulting from reactions initiated with R15 primers. (B) Repeating the experiment with the order of the dyes reversed. (C) Normalized scatter plot of co-hybridization of 200 pmol Cy5-labeled cDNA and 200 pmol Cy3-labeled cDNA, both resulting from reactions initiated with Inv primers. The central line is the median, and the upper and lower lines correspond to one $\log _{2}$-fold change up or down, respectively. Nonlinear normalization of the raw data was performed using Qspline (25).

detected on microarrays compared with cDNA generated by Inv/R6 priming.

Taken together, the results showed that R15 priming increases the amount of the resulting cDNA, but also yields cDNA with better representation of the original mRNA population. Although cDNA populations were generated for analysis with DNA microarrays as shown in this study, R15 primers could also be applied to single-cell real-time PCR with similar efficiency to Inv primers (data not shown), indicating that Inv primers can generally be substituted with R15 primers in other applications.

\section{DISCUSSION}

cDNA synthesis from RNA is a critical step in gene expression analysis, and the choice of priming can have large effects on the cDNA yield and quality as shown in this report. Priming with random pentadecamers resulted in $80 \%-90 \%$ of the complex aRNA or mRNA population being reverse transcribed. However, cDNA was measured spectroscopically after spin column purifications, a purification method that usually results in loss of DNA. It is therefore likely that R15 and $\mathrm{R} 12$ primer-initiated reactions have yields close to $100 \%$, indicating that almost the entire aRNA or mRNA pool (transcriptome) is reverse transcribed using R15 and R12 primers. The increased coverage of the transcription process using R 15 primers may explain why $55 \%-72 \%$ more genes were considered expressed using microarrays hybridized with R15-primed cDNA compared with Inv-primed cDNA.

Amplified RNA was used in these studies to have enough of a complex RNA population for all experiments and to avoid bias between the different cDNA pools (26). Because aRNA reactions are primed with poly(dT) primers, it is likely that this RNA population has a $3^{\prime}$ bias compared with mRNA, meaning that the aRNA is less complex than mRNA. The effect of using different length of primers was however similar if mRNA was used as template instead of aRNA (Figure 1A). In both cases, random pentadecamer priming resulted in close to $100 \%$ reverse transcription of the complex RNA sample, if losses during purification were accounted for. This indicates that the positive effects in hybridization reactions by using the R15 as primers in the cDNA synthesis on aRNA template most likely also apply to mRNA as template.

It is not clear why the use of R15 primers results in an increased yield of cDNA compared with R6 primers. One possible reason could be that shorter primers have difficulty annealing during the reverse transcription process. This can be deduced from calculating the melting temperature of random primers with different lengths and GC content (Supplementary Figure S2). It is likely that the R6 primers as well as the R15 primers hybridize during cooling on ice (see the Materials and Methods section for the procedure). However, preincubation at $20^{\circ} \mathrm{C}$ would induce the melting of AT-rich R6 primers, while AT-rich R15 primers will not melt (Supplementary Figure S2). It is less likely that R6 primers anneal to the RNA during synthesis at $42^{\circ} \mathrm{C}$, while annealing of GC-rich R15 primers can occur at $42^{\circ} \mathrm{C}$. It is therefore likely that $\mathrm{R} 15$ or longer primers will bind better to ATrich sequences than R6 primers during the reverse transcription procedure, indicating a mechanism for the observed increase in transcriptome coverage using longer primers. The lower yields obtained using random primers that were 18 nucleotides or longer can be the result of secondary structure formation or increased specificity resulting in fewer binding sites for the primers. This may indicate an optimum in terms of the length of random primers that is balanced between melting temperature of the primer-template duplex and the increased specificity originating from the increasing length.

Random pentadecamers have previously been reported to be used as primers in a preamplification step in whole genome amplification (WGA) (27). Presumably R15 primers were 
A

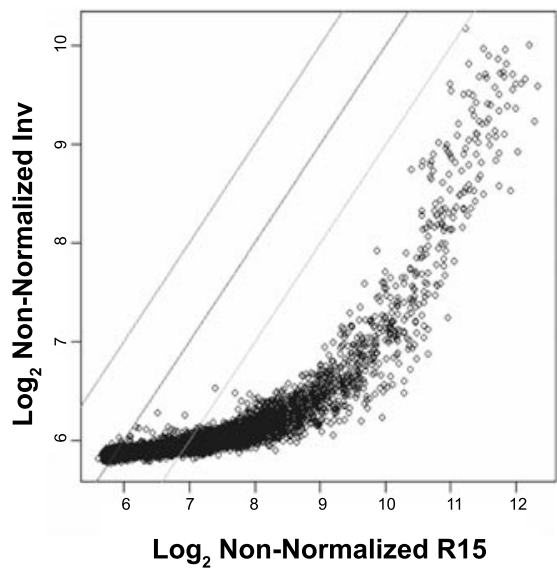

C

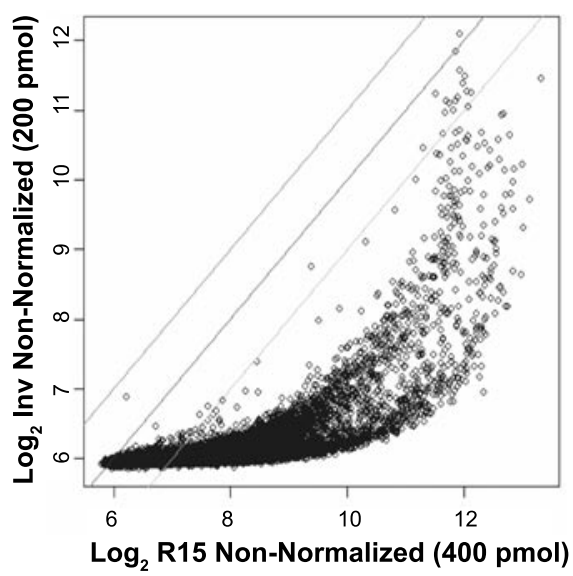

E

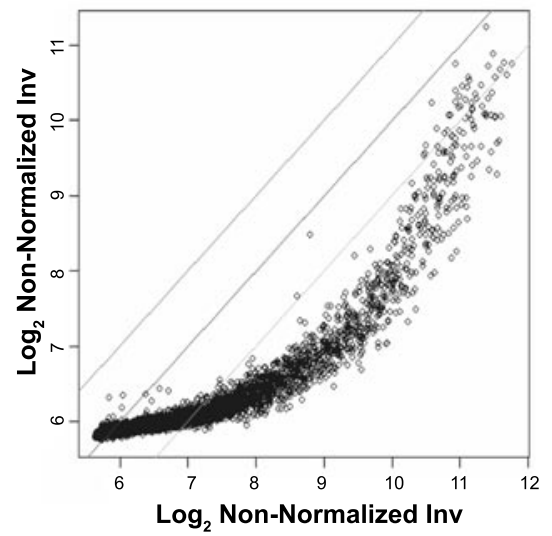

B
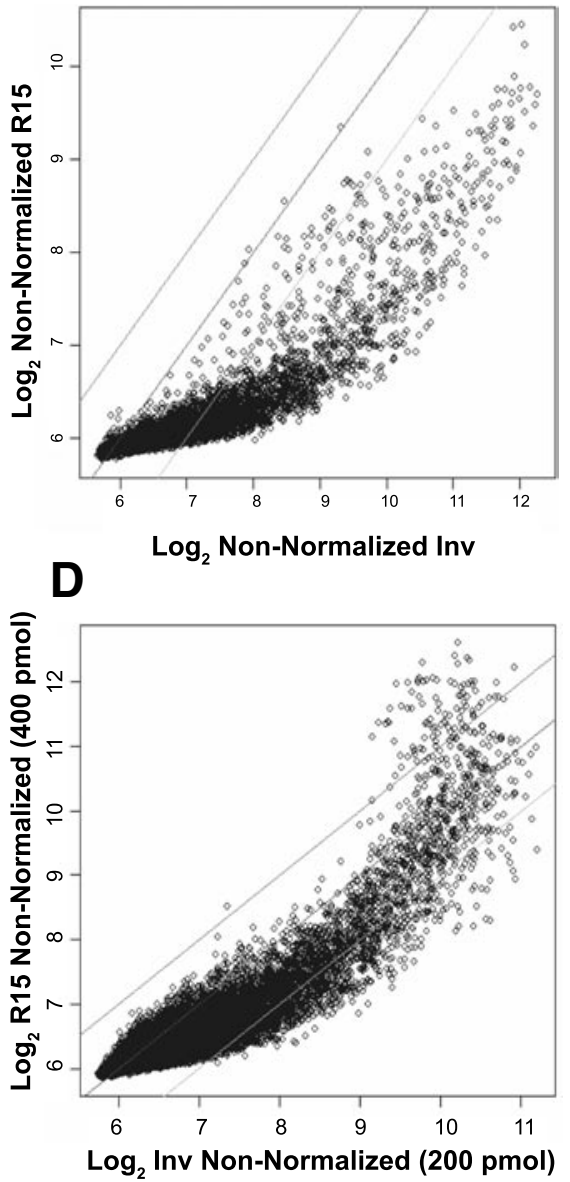

$\mathbf{F}$

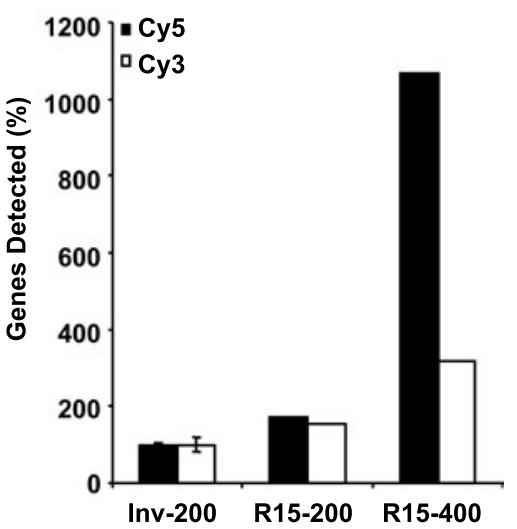

Figure 5. Scatter plots of non-normalized hybridizations to whole human transcriptome arrays. Cy5-labeled samples were always plotted on the y-axis. Molar amounts mentioned in pmol in this figure refer to the amount of Cy dye incorporated in the cDNA and not the amount of nucleotides of cDNA. (A, B, and E) Non-normalized data from Figure 4. (C) Repeating the experiment in panel A with twice the amount of labeled cDNA originating from R15-initiated reactions (400 pmol Cy dye-labeled cDNA) than Inv-initiated reactions (200 pmol Cy dye-labeled cDNA). (D) Repeating the experiment in panel $\mathrm{C}$ with the order of the dyes reversed. (F) Comparison of the amount of detectable genes with R15 and Inv priming. The data was normalized to the average amount of genes detected with 200 pmol of labeled cDNA primed with Inv primers in two independent hybridization reactions. To be able to compare the effects in the respective fluorescent channels each average, Cy3 and Cy5, respectively, was set to $100 \%$. A gene was considered detected if it displayed a signal that was $50 \%$ more than the background signal, calculated as the average of the 1000 weakest signals on the array.

chosen because they would function in the PCR where annealing was done at $37^{\circ} \mathrm{C}$, a temperature close to the $42^{\circ} \mathrm{C}$ used in reverse transcription reactions. Recently, reverse transcription polymerases operating at higher temperatures $\left(>50^{\circ} \mathrm{C}\right)$ have been introduced $(28,29)$. These enzymes should give better efficiency of reverse transcription reactions due to the fact that fewer secondary structures in the RNA template are encountered during the transcription process (29). It is likely that priming with longer primers could also have a greater positive effect on reverse transcription reactions performed at $50^{\circ} \mathrm{C}$, because a larger fraction of the longer primers will be able to form duplexes with the RNA template than the shorter primers.

It has previously been found that the requirement for an mRNA template can be reduced by a factor of 10 by using amino C6dT-modified random hexamers (22). It was speculated that the effect is most likely due to the increased incorporation of dyes in the target, because more amine groups are available on the target for the Cy dye esters to react with. However, if we assume that the cDNA is 400 nucleotides long on average, and the Nuc/Dye ratio is 40 , there are 10 dyes per cDNA copy. An additional dye from the C6dTmodified hexamer would only increase the dye content by $10 \%$ if the coupling efficiency is assumed to be $100 \%$. The relatively small gain in fluorescent signal that an amine group would give, according to this reasoning, was confirmed by our results in which an amino moiety linked to a C6 linker to R6 primers did not perform better than unmodified primers.

Techniques liker laser capture microdissection (LCM) enable the isolation of single cells (30). Comparing the gene expression profiles of single cells captured by LCM requires amplification (20), as the amount of mRNA in a single cell is limited. Linear amplification of RNA is usually performed utilizing a T7-based method (31). However, subsequent rounds of RNA amplification may include random hexamer or nonamer priming $(20,32)$. This technique yields only minute and highly precious amounts of genetic material. If the random hexamers 
or nonamers in these reactions were replaced with random pentadecamers, we believe, based on data presented in this work, that higher yields and quality of aRNA and subsequently better cDNA could be obtained. Other critical assays, like probing exon-arrays $(33,34)$ would also benefit from using R15 primers in reverse transcription, because a bias introduced by the patchy R6 priming could, in the worst case, significantly underestimate exon usage in cells.

In conclusion, a simple substitution of random hexamers with random pentadecamers in the reverse transcription reaction has many advantages, such as better genome coverage and a better yield. The effect is that better sensitivity is obtained using random pentadecamers as primers, which should be an advantage for gene expression analysis using microarrays or real-time PCR in general.

\section{ACKNOWLEDGMENTS}

The study was supported by The Danish Research Council [grant no. 2014-00-0003, Danish Biotetechnology Instrument Center (DABIC)]. The HeLa cell line was kindly donated by Dr. Peter Thomsen, Scandinavian Micro Biodevices (SMB), Farum, Denmark. We thank Jesper Petersen for critical reading of the manuscript.

\section{COMPETING INTERESTS STATEMENT}

The authors declare no competing interests.

\section{REFERENCES}

1. Aslanian, A., P.J. Iaquinta, R. Verona, and J.A. Lees. 2004. Repression of the Arf tumor suppressor by E2F3 is required for normal cell cycle kinetics. Genes Dev. 18:1413-1422.

2. DeRisi, J., L. Penland, P.O. Brown, M.L. Bittner, P.S. Meltzer, M. Ray, Y. Chen, Y.A. Su, and J.M. Trent. 1996. Use of a cDNA microarray to analyse gene expression patterns in human cancer. Nat. Genet. 14:457-460.

3. Marton, M.J., J.L. DeRisi, H.A. Bennett, V.R. Iyer, M.R. Meyer, C.J. Roberts, R. Stoughton, J. Burchard, et al. 1998. Drug target validation and identification of secondary drug target effects using DNA microarrays. Nat. Med. 4:1293-1301.
4.Maxfield, L.F., C.D. Fraize, and J.M. Coffin. 2005. From the cover: relationship between retroviral DNA-integration-site selection and host cell transcription. Proc. Natl. Acad. Sci. USA 102:1436-1441.

5. Yang, Y.H., S. Dudoit, P. Luu, D.M. Lin, V. Peng, J. Ngai, and T.P. Speed. 2002. Normalization for cDNA microarray data: a robust composite method addressing single and multiple slide systematic variation. Nucleic Acids Res. 30:e15.

6. Hughes, T.P., J. Kaeda, S. Branford, Z. Rudzki, A. Hochhaus, M.L. Hensley, I. Gathmann, A.E. Bolton, et al. 2003. Frequency of major molecular responses to imatinib or interferon alfa plus cytarabine in newly diagnosed chronic myeloid leukemia. N. Engl. J. Med. 349:1423-1432.

7. Melo, J.V., X.H. Yan, J. Diamond, F. Lin, N.C. Cross, and J.M. Goldman. 1996. Reverse transcription/polymerase chain reaction (RT/PCR) amplification of very small numbers of transcripts: the risk in misinterpreting negative results. Leukemia 10:1217-1221.

8. Asnafi, V., K. Beldjord, E. Boulanger, B. Comba, P. Le Tutour, M.-H. Estienne, F. Davi, J. Landman-Parker, et al. 2003. Analysis of TCR, pT $\alpha$, and RAG-1 in T-acute lymphoblastic leukemias improves understanding of early human T-lymphoid lineage commitment. Blood 101:2693-2703.

9. Bustin, S.A. and T. Nolan. 2004. Pitfalls of quantitative real-time reverse-transcription polymerase chain reaction. J. Biomol. Tech. 15:155-166.

10.Li, X., M. Miyajima, and H. Arai. 2005. Analysis of TGF- $\beta 2$ and TGF- $\beta 3$ expression in the hydrocephalic H-Tx rat brain. Childs Nerv. Syst. 21:32-38.

11. Mannhalter, C., D. Koizar, and G. Mitterbauer. 2000. Evaluation of RNA isolation methods and reference genes for RT-PCR analyses of rare target RNA. Clin. Chem. Lab. Med. 38:171-177.

12. Suzuki, S., M. Asamoto, K. Tsujimura, and T. Shirai. 2004. Specific differences in gene expression profile revealed by cDNA microarray analysis of glutathione S-transferase placental form (GST-P) immunohistochemically positive rat liver foci and surrounding tissue. Carcinogenesis 25:439-443.

13. Ståhlberg, A., J. Hakansson, X. Xian, H. Semb, and M. Kubista. 2004. Properties of the reverse transcription reaction in mRNA quantification. Clin. Chem. 50:509-515.

14. Brooks, E.M., L.G. Sheflin, and S.W. Spaulding. 1995. Secondary structure in the $3^{\prime}$ UTR of EGF and the choice of reverse transcriptases affect the detection of message diversity by RT-PCR. BioTechniques 19 : 806-815.

15. Michor, F., T.P. Hughes, Y. Iwasa, S. Branford, N.P. Shah, C.L. Sawyers, and M.A. Nowak. 2005. Dynamics of chronic myeloid leukaemia. Nature 435:1267-1270.

16. Brink, A.A., J.J. Oudejans, M. Jiwa, J.M. Walboomers, C.J. Meijer, and A.J. van den Brule. 1997. Multiprimed cDNA synthesis followed by PCR is the most suitable method for Epstein-Barr virus transcript analysis in small lymphoma biopsies. Mol. Cell. Probes 11:39-47.

17. Iturriza-Gomara, M., J. Green, D.W. Brown, U. Desselberger, and J.J. Gray. 1999. Comparison of specific and random priming in the reverse transcriptase polymerase chain reaction for genotyping group $\mathrm{A}$ rotaviruses. J. Virol. Methods 78:93-103.

18. Decraene, C., I. Reguigne-Arnould, C. Auffray, and G. Pietu. 1999. Reverse transcription in the presence of dideoxynucleotides to increase the sensitivity of expression monitoring with cDNA arrays. BioTechniques 27:962-966.

19. Schena, M., D. Shalon, R.W. Davis, and P.O. Brown. 1995. Quantitative monitoring of gene expression patterns with a complementary DNA microarray. Science 270:467-470.

20. Luo, L., R.C. Salunga, H. Guo, A. Bittner, K.C. Joy, J.E. Galindo, H. Xiao, K.E. Rogers, et al. 1999. Gene expression profiles of laser-captured adjacent neuronal subtypes. Nat. Med. 5:117-122.

21.Sterrenburg, E., R. Turk, J.M. Boer, G.B. van Ommen, and J.T. den Dunnen. 2002. A common reference for cDNA microarray hybridizations. Nucleic Acids Res. 30:e116.

22. Xiang, C.C., O.A. Kozhich, M. Chen, J.M. Inman, Q.N. Phan, Y. Chen, and M.J. Brownstein. 2002. Amine-modified random primers to label probes for DNA microarrays. Nat. Biotechnol. 20:738-742.

23. Yang, I.V., E. Chen, J.P. Hasseman, W. Liang, B.C. Frank, S. Wang, V. Sharov, A.I. Saeed, et al. 2002. Within the fold: assessing differential expression measures and reproducibility in microarray assays. Genome Biol. 3:research0062.

24. Kane, M.D., T.A. Jatkoe, C.R. Stumpf, J. Lu, J.D. Thomas, and S.J. Madore. 2000. Assessment of the sensitivity and specificity of oligonucleotide (50mer) microarrays. Nucleic Acids Res. 28:4552-4557.

25. Workman, C., L.J. Jensen, H. Jarmer, R. Berka, L. Gautier, H.B. Nielser, H.H. Saxild, C. Nielsen, et al. 2002. A new nonlinear normalization method for reducing variability in DNA microarray experiments. Genome Biol. 3:research0048.

26. Li, Y., T. Li, S. Liu, M. Qiu, Z. Han, Z. Jiang, R. Li, K. Ying, et al. 2004. Systematic comparison of the fidelity of aRNA, mRNA and T-RNA on gene expression profiling using cDNA microarray. J. Biotechnol. 107:19-28

27. Zhang, L., X. Cui, K. Schmitt, R. Hubert, W. Navidi, and N. Arnheim. 1992. Whole genome amplification from a single cell: implications for genetic analysis. Proc. Natl. Acad. Sci. USA 89:5847-5851.

28. Kawano, M., A.A. Reynolds, J. MirandaRios, and G. Storz. 2005. Detectionof $5^{\prime}$ - and 3'-UTR-derived small RNAs and cis-encoded antisense RNAs in Escherichia coli. Nucleic Acids Res. 33:1040-1050.

29. Zhang, Y.J., H.Y. Pan, and S.J. Gao. 2001 Reverse transcription slippage over the mRNA secondary structure of the LIP1 gene. BioTechniques 31:1286-1294.

30. Emmert-Buck, M.R., R.F. Bonner, P.D. Smith, R.F. Chuaqui, Z. Zhuang, S.R. Goldstein, R.A. Weiss, and L.A. Liotta. 1996. Laser capture microdissection. Science 274:998-1001.

31. Van Gelder, R.N., M.E. von Zastrow, A. Yool, W.C. Dement, J.D. Barchas, and J.H. Eberwine. 1990. Amplified RNA synthesized from limited quantities of heterogeneous cDNA. Proc. Natl. Acad. Sci. USA 87:1663-1667. 
32. Xiang, C.C., M. Chen, L. Ma, Q.N. Phan, J.M. Inman, O.A. Kozhich, and M.J. Brownstein. 2003. A new strategy to amplify degraded RNA from small tissue samples for microarray studies. Nucleic Acids Res. $31: \mathrm{e} 53$.

33. Dhami, P., A.J. Coffey, S. Abbs, J.R. Vermeesch, J.P. Dumanski, K.J. Woodward, R.M. Andrews, C. Langford, and D. Vetrie. 2005. Exon array CGH: detection of copy-number changes at the resolution of individual exons in the human genome. Am. J. Hum. Genet. 76:750-762.

34. Pinkel, D. and D.G. Albertson. 2005. Array comparative genomic hybridization and its applications in cancer. Nat. Genet. 37(Suppl): S11-S17.

Received 2 December 2006; accepted 15 February 2006.

Address correspondence to:

Martin Dufva

Department of Micro and Nanotechnology

Technical University of Denmark

Ørsteds Plads 345 east

DK-2800 Kgs, Lyngby, Denmark

e-mail:mdu@mic.dtu.dk 